\title{
GW200105 and GW200115 are compatible with a scenario of primordial black hole binary coalescences
}

\author{
Sai Wang ${ }^{1,2}$, Zhi-Chao Zhao ${ }^{3, \text { a }}$ \\ ${ }^{1}$ Theoretical Physics Division, Institute of High Energy Physics, Chinese Academy of Sciences, Beijing 100049, People's Republic of China \\ ${ }^{2}$ School of Physical Sciences, University of Chinese Academy of Sciences, Beijing 100049, People's Republic of China \\ ${ }^{3}$ Department of Astronomy, Beijing Normal University, Beijing 100875, People's Republic of China
}

Received: 19 July 2021 / Accepted: 30 December 2021 / Published online: 6 January 2022

(C) The Author(s) 2022

\begin{abstract}
Two gravitational wave events, i.e. GW200105 and GW200115, were observed by the Advanced LIGO and Virgo detectors recently. In this work, we show that they can be explained by a scenario of primordial black hole binaries that are formed in the early Universe. The merger rate predicted by such a scenario could be consistent with the one estimated from LIGO and Virgo, even if primordial black holes constitute a fraction of cold dark matter. The required abundance of primordial black holes is compatible with the existing upper limits from microlensing, caustic crossing and cosmic microwave background observations.
\end{abstract}

\section{Introduction}

Based on a second half of the third observing run, the LIGO Scientific and Virgo Collaborations (LVC) [1] reported two gravitational wave events, namely GW200105 and GW200115, which are compatible with neutron star-black hole (NSBH) binaries. At $90 \%$ confidence level, the primary components were found to be black holes with masses of $8.9_{-1.5}^{+1.2} M_{\odot}$ and $5.7_{-2.1}^{+1.8} M_{\odot}$, respectively, while the secondary ones $1.9_{-0.2}^{+0.3} M_{\odot}$ and $1.5_{-0.3}^{+0.7} M_{\odot}$, respectively. Compared with the maximal mass of neutron stars, both of the secondaries are compatible with neutron stars with a probability of $\sim 90 \%$. When the events were assumed to be representative of the entire NSBH population, the merger rate densities of GW200105 and GW200115 were inferred to be $16_{-14}^{+38} \mathrm{Gpc}^{-3}$ year $^{-1}$ and $36_{-30}^{+82} \mathrm{Gpc}^{-3}$ year $^{-1}$, respectively. When a broader distribution of component masses was assumed, they became $130_{-69}^{+112} \mathrm{Gpc}^{-3}$ year $^{-1}$. The above rates were found to be consistent with the model predictions of NSBH formation in isolated binaries or young star clusters. For either of the two events, however, LVC found no

a e-mail: zhaozc@bnu.edu.cn (corresponding author) evidence of tides or tidal disruption meanwhile identified no electromagnetic counterparts. Therefore, there is no direct evidence that the secondaries are neutron stars.

In this work, we show that a scenario of primordial black hole (PBH) binary coalescences can explain the origin of GW200105 and GW200115. Gravitational collapse of enhanced overdensities could produce PBHs in the early Universe [2-12]. PBHs can form binaries through several formation channels $[13,14]$. Amongst these channels, the most efficient one was originally proposed in Refs. [14,15], and recently extensively studied in Refs. [16-18] by considering such as a generic mass distribution of $\mathrm{PBHs}$ and the tidal forces from all the neighboring PBHs and linear density perturbations. To determine if GW200105 and GW200115 are primordial, we have to check whether their merger rate densities can be accounted for with the corresponding PBH abundance allowed by the existing upper limits (see review in Ref. [19] and references therein). In the following, we will compute the merger rate density of PBHs in two specific models by following Ref. [20]. The first one assumes that the mass function is determined by GW200105 and GW200115. The second one assumes that all the black hole binary coalescences observed by LVC are primordial. The predicted rates will be compared with the estimated ones from LVC.

The rest of the paper is arranged as follows. In Sect. 2, we show the formula for the merger rate of PBH binaries. In Sect. 3, we briefly review the log-normal mass function of PBHs. Our results and conclusions are shown in Sects. 4 and 5 , respectively.

\section{Merger rate of PBH binaries}

We consider the formation channel of PBH binaries in the early Universe [14]. It is known that this channel makes a 
dominant contribution to the $\mathrm{PBH}$ merger rate [17]. This means neglects of the binary formation mechanisms, such as dynamical captures and three-body interactions, in the late Universe [21]. The late-Universe formation mechanism was originally proposed in Ref. [14] and revisited recently in Ref. [15]. Two neighboring PBHs could form a binary due to torque from a third neighboring $\mathrm{PBH}$, and merge with each other within the age of the Universe due to the energy loss via gravitational emissions. However, the mass distribution of PBHs was assumed to be monochromatic in the original literature. More recently, the merger rate of PBHs with an extended mass function was studied in Refs. [16-18]. To be specific, Ref. [16] took into account the tidal force from a $\mathrm{PBH}$ which is closest to the center of mass of PBH binary. Reference [17] assumed a flat mass function of PBHs within a narrow mass range. To generalize the above two works, Ref. [18] took into account the tidal forces from all neighboring PBHs and linear density perturbations, and meanwhile considered a generic mass function of PBHs. Therefore, we follow Ref. [18] to compute the merger rate of $\mathrm{PBH}$ binaries with a log-normal mass distribution.

For PBHs within mass intervals of $\left(m_{i}, m_{i}+d m_{i}\right)$ and $\left(m_{j}, m_{j}+d m_{j}\right)$, the merger rate per unit volume within temporal interval $(t, t+d t)$ is defined as $R(t)=$ $\mathcal{R}\left(m_{i}, m_{j}, t\right) d m_{i} d m_{j}$ in units of $\mathrm{Gpc}^{-3}$ year $^{-1}$. The comoving merger rate density is obtained as follows [18]

$$
\begin{aligned}
\mathcal{R}\left(m_{i}, m_{j}, t\right) & \\
= & 3.9 \times 10^{6} \times\left(\frac{t}{\tau}\right)^{-\frac{34}{37}} f^{2}\left(f^{2}+\sigma_{\mathrm{eq}}^{2}\right)^{-\frac{21}{74}} \min \left(\frac{P\left(m_{i}\right)}{m_{i}}, \frac{P\left(m_{j}\right)}{m_{j}}\right) \\
& \times\left(\frac{P\left(m_{i}\right)}{m_{i}}+\frac{P\left(m_{j}\right)}{m_{j}}\right)\left(m_{i} m_{j}\right)^{\frac{3}{37}}\left(m_{i}+m_{j}\right)^{\frac{36}{37}},
\end{aligned}
$$

where $f=\Omega_{\mathrm{pbh}} / \Omega_{\mathrm{m}}$ is the total fraction of non-relativistic matter in PBHs, $\sigma_{\text {eq }} \approx 0.005$ is the variance of overdensities of the rest of dark matter on scales of order $\mathcal{O}\left(10^{-2}-\right.$ $\left.10^{5}\right) M_{\odot}$ at equality [17], $\min \left(x_{1}, x_{2}\right)$ selects the minimal value between $x_{1}$ and $x_{2}, P(m)$ is the mass function of PBHs, $t$ and $\tau$ denote the cosmic time and the present age of the universe, respectively. The abundance of PBHs in dark matter is $f_{\mathrm{pbh}}=\Omega_{\mathrm{pbh}} / \Omega_{\mathrm{dm}} \simeq f \Omega_{\mathrm{m}} / \Omega_{\mathrm{dm}}$. Here, $\Omega_{\mathrm{pbh}}, \Omega_{\mathrm{dm}}$ and $\Omega_{\mathrm{m}}$ denote the energy density fractions of PBHs, dark matter and non-relativistic matter, respectively, in the critical energy density of the Universe at present. In addition, we would not consider the redshift evolution of the merger rate density, since only the low-redshift events are detectable for LVC. Throughout this work, we fix all cosmological parameters to be the best-fit values from the Planck 2018 results [22].

\section{Mass function of PBHs}

The mass function of PBHs can be determined by the production mechanisms of PBHs in the early Universe. The most likely one is based on the gravitational collapse of overdensities in the radiation dominated epoch of the Universe [28]. Therefore, the mass function of PBHs depends on the properties of primordial curvature perturbations. It is usually parametrized to be a log-normal function as follows

$P(m)=\frac{1}{\sqrt{2 \pi} \sigma m} \exp \left(-\frac{\ln ^{2}\left(m / m_{*}\right)}{2 \sigma^{2}}\right)$,

where $m_{*}$ and $\sigma$ stand for the center mass and width, respectively $[18,20,23]$. In this work, we consider two different methods to determine values of $m_{*}$ and $\sigma$. First, we assume that all observed black hole binaries are primordial. The results have been shown to be $m_{*}=19 M_{\odot}$ and $\sigma=0.97$ [20], which will be used in this work. There are also alternative choices of mass function [18], which would not significantly change our results of the abundance of PBHs. Second, we assume that only GW200105 and GW200115 are of primordial origin and they determine the mass function of PBHs. Typically, we choose one half of the total mass to determine the value of $m_{*}$, namely $m_{*}=5.4 M_{\odot}$ for GW200105 and $m_{*}=3.6 M_{\odot}$ for GW200115. Meanwhile, we set the width to be $\sigma=m_{*} / 10 M_{\odot}$, implying $\sigma=0.54$ for GW200105 and $\sigma=0.36$ for GW200115. In such a case, slightly different choices would change our predictions on the merger rate density by a factor of $\mathcal{O}(1)$. Based on Eq. (1), they would not significantly alter our results of the abundance of PBHs [20].

\section{Limits on PBH abundance}

Our results are shown in Fig. 1. We depict the limits on $f_{\mathrm{pbh}}$ estimated from the merger rates of GW200105 (red triangle) and GW200115 (green triangle), and the broad mass function (purple circle), respectively. The gray solid lines denote the error bars at $90 \%$ CL, due to uncertainties in the measurements of the merger rates [1]. The dotted curves stand for the existing upper limits at 90\% CL from OGLE (blue) [24], EROS/MACHO (cyan) [25,26], ICARUS (purple) [27], and Planck (orange) [28], which are shown here for comparison.

In the case of single PBH event, the mass function is determined by the considered event, i.e. GW200105 or GW200115. Given the observational uncertainty, we derive the allowed abundance of PBHs which matches the observed merger rate of the event [1]. We find that both of the events are well explained by such a scenario, when we take $f_{\mathrm{pbh}} \simeq$ $\mathcal{O}\left(10^{-2}\right)$ that is compatible with the existing best constraint $f_{\mathrm{pbh}} \simeq \mathcal{O}\left(10^{-1}\right)$ from caustic crossing [27]. We also find that the inferred upper limits are close to the existing upper limit, implying that such a scenario may be further tested 
Fig. 1 Constraints on $f_{\text {pbh }}$ with $90 \%$ CL error bars (gray solid) inferred from the merger rates for GW200105 (red triangle) and GW200115 (green triangle), and the broad mass function (purple circle). The existing upper limits on $f_{\mathrm{pbh}}$ at $90 \% \mathrm{CL}$ from OGLE (blue), EROS/MACHO (cyan), ICARUS (purple), and Planck (orange) are shown for comparison

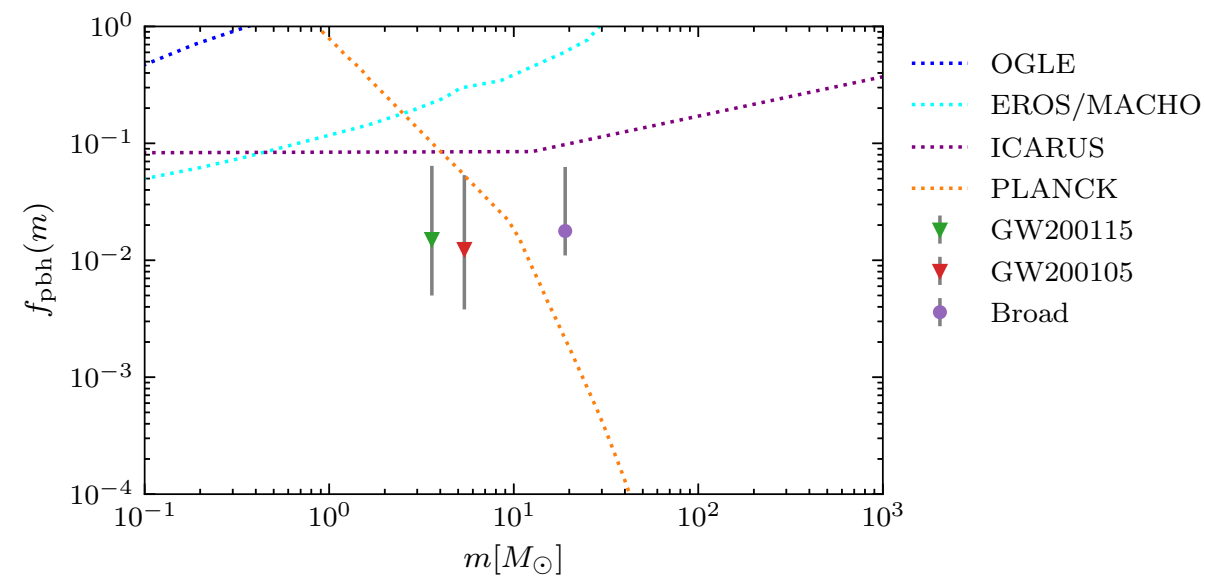

in the near future. Furthermore, it is interesting to find that the mass function with $m_{*}=5.4 M_{\odot}$ and $\sigma=0.54$ can almost precisely explain GW200105 and GW200115 simultaneously. But this coincidence may be accidental.

In the case of the broad mass distribution, the mass function is determined by all the observed black hole binaries, since all of them are assumed to be primordial. This has been done by using maximum-likelihood analysis in an existing literature [20]. In our work, the merger rate is predicted via integration over $m_{i} \in[2.5,40] M_{\odot}$ and $m_{j} \in[1,3] M_{\odot}$, following a method consistent with Ref. [1]. Given $f_{\mathrm{pbh}} \simeq$ $\mathcal{O}\left(10^{-2}\right)$, we conclude that the merger rates predicted by this model are compatible with those of GW200105 and GW200115 reported by LVC [1], although the predicted constraint on $f_{\text {pbh }}$ is in tension with that inferred from the cosmic microwave background measured by Planck [28]. In addition, by combining the broad mass function from Ref. [20] with the two recently observed events, i.e. GW200105 and GW200115, we obtain a joint result of $m_{*}=16.4 M_{\odot}$ and $\sigma=1.0$, which is slightly different with that of Ref. [20].

\section{Conclusions}

In this work, we have shown that the scenario of PBH binary coalescences can account for the observed merger rates of GW200105 and GW200115. We computed the merger rates in two different models. The first one assumed that only GW200105 and GW200115 have a primordial origin. The second one assumed that all the observed black hole binaries are primordial. Given $f_{\mathrm{pbh}} \simeq \mathcal{O}\left(10^{-2}\right)$, we found that both of the two models can explain the recently reported events. For the former, the required abundance of PBHs were found to be compatible with the existing upper limits from other observations. The inferred upper limits were shown to be close to the existing upper limits, implying that such scenarios can be further tested by observations of (SGWB) in the near future [29-33]. However, the latter was found to be in tension with the exiting observational limits. In addition, we found that the log-normal mass function of PBHs with $m_{*}=5.4 M_{\odot}$ and $\sigma=0.54$ almost simultaneously explains the observed merger rates of GW200105 and GW200115, including the center values and error bars. In summary, depending on the abundance of PBHs, the scenario of PBH binaries formed through the early-Universe channel could well explain GW200105 and GW200115 events.

Acknowledgements We would like to acknowledge Prof. Dr. Zhoujian Cao and Dr. Zucheng Chen for helpful discussions. S.W. is supported by the grants from the National Natural Science Foundation of China with Grant NO. 12175243, the Institute of High Energy Physics with Grant NO. Y954040101, the Key Research Program of the Chinese Academy of Sciences with Grant NO. XDPB15, and the science research grants from the China Manned Space Project with NO. CMS-CSST-2021B01. Z.C.Z. is supported by the National Natural Science Foundation of China with Grant No. 12005016.

Data Availability Statement This manuscript has no associated data or the data will not be deposited. [Authors' comment: The data is deposited in the text.]

Open Access This article is licensed under a Creative Commons Attribution 4.0 International License, which permits use, sharing, adaptation, distribution and reproduction in any medium or format, as long as you give appropriate credit to the original author(s) and the source, provide a link to the Creative Commons licence, and indicate if changes were made. The images or other third party material in this article are included in the article's Creative Commons licence, unless indicated otherwise in a credit line to the material. If material is not included in the article's Creative Commons licence and your intended use is not permitted by statutory regulation or exceeds the permitted use, you will need to obtain permission directly from the copyright holder. To view a copy of this licence, visit http://creativecomm ons.org/licenses/by/4.0/.

Funded by $\mathrm{SCOAP}^{3}$.

\section{References}

1. R. Abbott et al., Observation of gravitational waves from two neutron star-black hole coalescences. Astrophys. J. Lett. 915, L5 (2021) 
2. S. Hawking, Gravitationally collapsed objects of very low mass. Mon. Not. R. Astron. Soc. 152, 75 (1971)

3. B.J. Carr, S.W. Hawking, Black holes in the early Universe. Mon. Not. R. Astron. Soc. 168, 399-415 (1974)

4. J. Garcia-Bellido, A.D. Linde, D. Wands, Density perturbations and black hole formation in hybrid inflation. Phys. Rev. D 54, 60406058 (1996)

5. S. Clesse, J. Garcia-Bellido, Massive primordial black holes from hybrid inflation as dark matter and the seeds of galaxies. Phys. Rev. D 92, 023524 (2015)

6. A.D. Dolgov, S.I. Blinnikov, Stars and black holes from the very early Universe. Phys. Rev. D 89(2), 021301 (2014)

7. T. Harada, C.-M. Yoo, K. Kohri. Threshold of primordial black hole formation. Phys. Rev. D 88(8), 084051 (2013) (Erratum: Phys. Rev. D 89(2), 029903 (2014))

8. T. Harada, C.-M. Yoo, K. Kohri, K. Nakao, S. Jhingan, Primordial black hole formation in the matter-dominated phase of the Universe. Astrophys. J. 833(1), 61 (2016)

9. M.Y. Khlopov, Primordial black holes. Res. Astron. Astrophys. 10, 495-528 (2010)

10. K.M. Belotsky, A.D. Dmitriev, E.A. Esipova, V.A. Gani, A.V. Grobov, M.Y. Khlopov, A.A. Kirillov, S.G. Rubin, I.V. Svadkovsky, Signatures of primordial black hole dark matter. Mod. Phys. Lett. A 29(37), 1440005 (2014)

11. S.V. Ketov, M.Y. Khlopov, Cosmological probes of supersymmetric field theory models at superhigh energy scales. Symmetry 11(4), $511(2019)$

12. Z. Zhou, J. Jiang, Y.-F. Cai, M. Sasaki, S. Pi, Primordial black holes and gravitational waves from resonant amplification during inflation. Phys. Rev. D 102(10), 103527 (2020)

13. S. Bird, I. Cholis, J.B. Munoz, Y. Ali-Haimoud, M. Kamionkowski, E.D. Kovetz, A. Raccanelli, A.G. Riess, Did LIGO detect dark matter? Phys. Rev. Lett. 116(20), 201301 (2016)

14. T. Nakamura, M. Sasaki, T. Tanaka, K.S. Thorne, Gravitational waves from coalescing black hole MACHO binaries. Astrophys. J. Lett. 487, L139-L142 (1997)

15. M. Sasaki, T. Suyama, T. Tanaka, S. Yokoyama, Primordial black hole scenario for the gravitational-wave event GW150914. Phys. Rev. Lett. 117(6), 061101 (2016)

16. M. Raidal, V. Vaskonen, H. Veermäe, Gravitational Waves from Primordial Black Hole Mergers. JCAP 09, 037 (2017)

17. Y. Ali-Haïmoud, E.D. Kovetz, M. Kamionkowski, Merger rate of primordial black-hole binaries. Phys. Rev. D 96(12), 123523 (2017)

18. Z.-C. Chen, Q.-G. Huang, Merger Rate Distribution of PrimordialBlack-Hole Binaries. Astrophys. J. 864(1), 61 (2018)

19. B. Carr, K. Kohri, Y. Sendouda, J. Yokoyama, Constraints on primordial black holes (2020)
20. V. De Luca, V. Desjacques, G. Franciolini, P. Pani, A. Riotto, GW190521 mass gap event and the primordial black hole scenario. Phys. Rev. Lett. 126(5), 051101 (2021)

21. K. Kritos, V. De Luca, G. Franciolini, A. Kehagias, A. Riotto, The astro-primordial black hole merger rates: a reappraisal. JCAP $\mathbf{0 5}$, 039 (2021)

22. N. Aghanim et al., Planck 2018 results. VI. Cosmological parameters. Astron. Astrophys. 641, A6 (2020)

23. B. Carr, M. Raidal, T. Tenkanen, V. Vaskonen, H. Veermäe, Primordial black hole constraints for extended mass functions. Phys. Rev. D 96(2), 023514 (2017)

24. H. Niikura, M. Takada, S. Yokoyama, T. Sumi, S. Masaki, Constraints on earth-mass primordial black holes from OGLE 5-year microlensing events. Phys. Rev. D 99(8), 083503 (2019)

25. P. Tisserand et al., Limits on the Macho Content of the galactic halo from the EROS-2 survey of the magellanic clouds. Astron. Astrophys. 469, 387-404 (2007)

26. R.A. Allsman et al., MACHO project limits on black hole dark matter in the 1-30 solar mass range. Astrophys. J. Lett. 550, L169 (2001)

27. M. Oguri, J.M. Diego, N. Kaiser, P.L. Kelly, T. Broadhurst, Understanding caustic crossings in giant arcs: characteristic scales, event rates, and constraints on compact dark matter. Phys. Rev. D 97(2), 023518 (2018)

28. P.D. Serpico, V. Poulin, D. Inman, K. Kohri, Cosmic microwave background bounds on primordial black holes including dark matter halo accretion. Phys. Rev. Res. 2(2), 023204 (2020)

29. S. Wang, Y.-F. Wang, Q.-G. Huang, T.G.F. Li, Constraints on the primordial black hole abundance from the first advanced LIGO observation run using the stochastic gravitational-wave background. Phys. Rev. Lett. 120(19), 191102 (2018)

30. S. Wang, T. Terada, K. Kohri. Prospective constraints on the primordial black hole abundance from the stochastic gravitational-wave backgrounds produced by coalescing events and curvature perturbations. Phys. Rev. D 99(10), 103531 (2019) (erratum: Phys. Rev. D 101(6), 069901 (2020))

31. K. Kohri, T. Terada, Solar-mass primordial black holes explain NANOGrav hint of gravitational waves. Phys. Lett. B 813, 136040 (2021)

32. S. Mukherjee, J. Silk, Can we distinguish astrophysical from primordial black holes via the stochastic gravitational wave background? 5 (2021)

33. V. De Luca, G. Franciolini, P. Pani, A. Riotto. The minimum testable abundance of primordial black holes at future gravitationalwave detectors. 6 (2021) 